\title{
SENSIBILIDAD DE GÉNERO EN LA INVESTIGACIÓN SOBRE POLÍTICAS SANITARIAS
}

\author{
ERICA BRIONES VOZMEDIANO \\ Universidad de Alicante
}

Recibido/15/07/2011

Aceptado/25/09/2011

\begin{abstract}
Referencia del artículo comentado
PEIRÓ PÉREZ, Rosana, ÁlVAREZ-DARDET, Carlos, COLOMER Concha, MOYA Carmela, BORRELL Carmen, PLASENCIA Antonio, ZAFRA Eduardo y PASARÍN María Isabel. «Sensibilidad de género en la formulación de planes de saludo en España. Lo que pudo ser y no fue». Gaceta Sanitaria, 18, (2004), pp. 36-46.
\end{abstract}

\section{Resumen}

Objetivo: Examinar de forma sistemática la sensibilidad de género en las políticas formuladas en los planes de salud, de las comunidades autónomas (CCAA) de España que lo tienen disponible. Definimos «sensibilidad de género» como el grado en que los planes de salud tienen en cuenta la existencia del género como categoría de relación y desarrollan acciones para aminorar las desigualdades debidas a éste.

Material y métodos: Se diseñó un cuestionario con una estructura similar a la de los planes de salud, que a su vez están basados en la propuesta de la Organización Mundial de la Salud (OMS). Éstos tienen una parte introductoria, que hemos denominado de «contenido simbólico» y describe objetivos y valores generales del plan de salud, y otra, que hemos denominado el «contenido operativo» del plan de salud, donde se concretan los objetivos específicos y las intervenciones para conseguirlos. Se construyen índices de sensibilidad simbólica de género y de sensibilidad operativa de género. Resultados: Se analizaron 13 planes de salud. El País Vasco, la Comunidad Valenciana y Canarias tienen un alto índice de sensibilidad simbólica que no se corresponde en todas las comunidades con su índice de sensibilidad operativa. El País Vasco, Cataluña y Galicia tienen los índices de sensibilidad operativa de género más altos. 
Conclusiones: La sensibilidad de género en los planes de salud es muy desigual por CCAA. Algunas vías para la acción serían la formación de coaliciones y redes de profesionales y colectivos para poner de manifiesto el problema, así como construir sobre la experiencia de las CCAA con mayor sensibilidad de género en sus propuestas.

Palabras clave: Plan de salud. Desigualdad. Sensibilidad de género. Política sanitaria. 


\section{Introducción}

El artículo de Peiró y colaboradores, ofrece un ejemplo de métodos útiles de investigación con sensibilidad de género. En él se examina de forma sistemática la sensibilidad de género en los planes de salud de las Comunidades Autónomas (CCAA) españolas, entendida como la medida en que estas políticas sanitarias tienen en cuenta la existencia del género como categoría de relación y desarrollan acciones para aminorar las desigualdades debidas al género.

La metodología empleada resulta novedosa, a la vez que práctica, para la investigación en salud pública. La/os autora/es describen y comparan los diferentes planes de salud de las CCAA, para lo que diseñaron un cuestionario ad hoc como guía de lectura que recogía información sobre la sensibilidad de género de cada uno de los apartados de los planes de salud. Éstos se estructuraban en una parte introductoria, que describía los objetivos y valores generales del plan de salud y se denominó «contenido simbólico», y otra dónde se concretaban los objetivos específicos y las intervenciones para conseguirlos, que se denominó «contenido operativo». Se construyeron índices de sensibilidad de género simbólica y operativa, en base al número de respuestas positivas a las preguntas dicotómicas que planteaba el cuestionario

En este estudio se analizaron 13 planes de salud de las CCAA que lo tenían disponible. Los aspectos que se analizan de estos planes de salud son los problemas de salud priorizados, los entornos para la salud -entendidos como el lugar y el contexto social en que las personas desarrollan las actividades diarias y en el cual interactúan factores ambientales, organizativos y personales que afectan a la salud y el bienestar ${ }^{1}$ - los objetivos de apoyo al desarrollo del plan y la existencia de capítulos específicos sobre salud reproductiva, salud de las mujeres y grupos de población vulnerable.

El plan de salud del País Vasco resultó ser el más sensible al género y el problema de salud para el que se proponían más intervenciones fue la enfermedad oncológica, debido a la especificación de intervenciones en cánceres

1. ORGANIZACIÓN MUNDIAL DE LA SALUD: Promoción de la salud. Glosario. Ginebra: Organización Mundial de la Salud; 1998.

Feminismo/s 18, diciembre 2011, pp. 343-351 
ligados al sexo. Se concluyó que la sensibilidad de género en los planes de salud era muy desigual por CCAA y los hallazgos sugirieron que la integración del género en los planes de salud de las CCAA aún era escasa.

\section{Desarrollo}

Desde 1995, a partir de IV Conferencia Mundial sobre la Mujer de Beijing, la ONU insta a diseñar, implementar y supervisar políticas y programas que sean efectivos, eficientes y sensibles al género. Además, incorporar el género de manera transversal en las políticas sanitarias es una de las recomendaciones de la OMS, que en 2002 aprobó su Política de Género con el objetivo de contribuir a la mejora de la salud tanto de mujeres como de hombres, mediante investigaciones, políticas y programas de salud que presten la debida atención a las cuestiones de género y que promuevan la equidad y la igualdad entre hombres y mujeres ${ }^{2}$.

Los métodos de investigación con perspectiva de género constituyen herramientas que posibilitan el mainstreaming de género, una estrategia política y técnica para avanzar en la equidad de género, uno de los principios básicos de justicia social. Aplicar el mainstreaming de género implica que se reconozcan las necesidades de mujeres y hombres y las desigualdades de género en toda la estructura de las políticas públicas, por lo que transversalizar la perspectiva de género suele implicar la renovación de las políticas en todas las fases del proceso: diseño, implementación y evaluación ${ }^{3}$.

Los programas y políticas sanitarias sensibles al género son necesarias porque tienen la capacidad de transformar las relaciones de género, si promueven el empoderamiento de las mujeres, fomentan la conciencia crítica de roles y normas de género, se dirigen a cambiar las relaciones desiguales de poder o la distribución de recursos. Por ejemplo, la integración del género en los programas de salud reproductiva tiene efectos positivos sobre los resultados en este ámbito ${ }^{4}$. Si las políticas que promueven la igualdad de género son diseñadas con el fin de ser implementadas, se requiere una identificación rigurosa de los aspectos en común y las diferencias entre las necesidades en salud de

2. ORGANIZACIÓN MUNDIAL DE LA SALUD: Integrating gender perspectives in the work of WHO. WHO gender policy. Ginebra,: Organización Mundial de la Salud; 2002.

3. Peiró, Rosana, Vives, Carmen, Álvarez-Dardet, Carlos y R. MAS: «El Análisis De Políticas Con Enfoque De Género Y Salud.», en Carmen Borrell y Lucía ArTAZCoz (ed.), $5^{a}$ Monografía. Sociedad Española De Epidemiología. Investigación Sobre Género Y Salud, Barcelona, Sociedad Española de Epidemiología, 2007, pp. 119-37.

4. ORGANIZACIÓN MUNDIAL DE LA SALUD, A summary of the "so what? Report, Ginebra, Organización Mundial de la Salud, 2005. 
mujeres y hombres, así como un análisis de los obstáculos para acceder a los servicios sanitarios asociados al género ${ }^{5}$.

En este breve ensayo se plantea por qué es necesario analizar las políticas públicas desde el enfoque de género. Las políticas sociales impactan de manera diferente en mujeres y hombres y, como consecuencia, pueden producir o incrementar las desigualdades en salud si ignoran el género o están destinadas a proteger a uno de los dos sexos. Debido, de alguna manera, al patrón androcéntrico de la ciencia, se tiende a diseñar políticas e intervenciones centradas en el modelo masculino, obviando en ocasiones las necesidades de las mujeres $^{6}$.

Aunque la equidad de género es un objetivo que cada vez se está incluyendo con más frecuencia en las políticas sanitarias, es necesario evaluar si los objetivos van seguidos de acciones para implementarlos. Según Peiró y cols, aunque el análisis realizado no comprenda las intervenciones que realmente se implantan, el análisis de las intenciones explícitas recogidas en los planes de salud puede informar sobre los ejes en los que se mueven estas políticas, que con posterioridad inspirarán las acciones que se emprendan. Mediante el análisis de género se podrán identificar buenas prácticas para ofrecer una atención sanitaria equitativa y prevenir las desigualdades en salud entre hombres y mujeres ${ }^{7,8}$. Además, aplicar el enfoque de género al investigar transmite la evidencia existente sobre el impacto de género en el acceso a los servicios de salud o en la atención a determinados problemas de salud y, a la vez, supone un esfuerzo para salvar la distancia entre los responsables de políticas de salud y los investigadores en género y salud.

Siguiendo los principios rectores de la nueva política global de salud de la OMS «Salud para todos en el siglo XXI», que plantea la igualdad de género como uno de los principios básicos, el cuestionario diseñado en este estudio

5. Gispert, Rosa: «Objetivo 33: Desarrollo De Las Políticas Salud Para Todos.», en ÁlvAREZ-DARDET, Carlos, y PEIRÓ, Rosana (eds.) Informe SESPAS 2000.

6. Ruiz Cantero, Maria Teresa, Vives Cases, Carmen, Artazcoz, Lucía, Delgado, Ana, García Calvente Maria del Mar, Miqueo, Consuelo, Montero, Isabel, Ortiz, Rocío, RONDA, Elena, RUIZ, Isabel, VALLS, Carme «A framework to analyse gender bias in epidemiological research», Journal of Epidemiology Community Health, 61, (2007), pp. 46-53.

7. Peiró, Rosana, Alvarez-Dardet, Carlos, Plasencia, Antoni, Borrell, Carmen, ColoMER, Concha, MOYA, Carmela, PASARín, Maria Isabel, ZAFrA, Eduardo. «Rapid appraisal methodology for 'health for all' policy formulation analysis», Health Policy, 62:3,.(2002), pp. 309-28.

8. Colomer-Revuelta, Concha, Peiró-Pérez, Rosana, López-Rodríguez, Rosa M., EspiGA-López Isabel, SÁiz-MARTíENZA-ACitoreS, Isabel, SORIANO-Villarroel, Isabel. «Policies, politics and gender research». Journal of Epidemiology and Community Health, 61, (2007), pp. iil-ii2.

Feminismo/s 18, diciembre 2011, pp. 343-351 
exploró si se tenía en cuenta la igualdad de género como un objetivo o valor de las políticas sanitarias, si se ofrecían datos desagregados por sexo, si se describían situaciones de salud diferentes entre hombres y mujeres -y, en caso afirmativo, si se incluían las intervenciones adecuadas para cada una-, o si se contemplaba la participación de sectores que trabajan con la igualdad de género, entre otros aspectos. Además, la metodología empleada se adecúa a las recomendaciones de la OMS sobre las herramientas de género propuestas dentro del proceso de creación de políticas, que son: obtener datos desagregados por sexo, efectuar un análisis cuantitativo y cualitativo y basarse en el ciclo de vida de las personas, identificar información prioritaria en las políticas e identificar acciones del sector salud ${ }^{9}$.

Esta metodología resulta útil para ser aplicada en posteriores estudios con un diseño similar. Existen estudios posteriores que analizan políticas sanitarias desde la perspectiva de género ${ }^{10}$ y que incluso se han basado en esta experiencia, al realizar un diagnóstico del grado de sensibilidad de género de los planes de salud del contexto internacional ${ }^{11}$. La propuesta de este ensayo es que, para que el análisis de políticas operativamente fuera más útil, el protocolo a aplicar debería comprobar si las políticas incorporan de manera transversal:

- el género como principio o valor

- contenidos de equidad de género

- las diferencias de género como determinante de las desigualdades en salud

- la atención al género como elemento en situaciones de discriminaciones múltiples

- la consulta a órganos consultivos específicos en materia de género para la formulación de las políticas.

El protocolo empleado por Peiró y cols. también comprueba si los planes incluyen apartados específicos sobre salud reproductiva o salud de las mujeres.

9. ORGANIZACIÓN MUNDIAL DE LA SALUD: WHA 60.25 Resolution. Strategy for integrating gender analysis and actions into the work of WHO. Ginebra, Organización Mundial de la Salud, 2007.

10. Stewart, Donna E., Linda M. Dorado, Natalia Diaz-Granados, Marta Rondon, Javier SAAVEDRA, Jose POSADA-VILla y Yolanda TORRES. «Examining Gender Equity in Health Policies in a Low- (Peru), Middle- (Colombia), and High- (Canada) Income Country in the Americas.», Journal of Public Health Policy, 30: 4, (2009), pp. 439-54.

11. Briones Vozmediano, Erica, Vives Cases, Carmen, Peiró Pérez, Rosana «Sensibilidad de género en los planes nacionales de salud del contexto internacional», Informe SESPAS 2009. 
La existencia de estos apartados sugiere que los hombres son representados como el prototipo de la normalidad y, por tanto, las mujeres como la desviación, quedando invisibilizadas y limitando su salud y morbilidad a su función reproductiva. Se hace necesario promover una comprensión de la salud de las mujeres más amplia y comprobar si las políticas sanitarias incluyen entre sus prioridades otros aspectos de morbilidad más prevalentes en las mujeres (por ejemplo, problemas de salud emergentes como la fibromialgia, fatiga crónica y sensibilidad química múltiple) o la desigualdad en el acceso a los servicios sanitarios. Hay que tener en cuenta que el paso previo a la formulación de las políticas sobre un tema determinado es su construcción social como problema, impulsada por un colectivo con capacidad de influencia sobre la opinión pública, como el movimiento de mujeres, y con autoridad suficiente como para emprender acciones en respuesta a dichos problema ${ }^{12,13}$.

\section{Conclusión}

Este artículo subraya el género como un determinante social de la salud y, por tanto, que las desigualdades de género impactan en las desigualdades de salud. Es ampliamente reconocido que las desigualdades de género son uno de los factores limitantes de la capacidad de mujeres y hombres para desarrollar plenamente su potencial de salud, por lo que atender a las diferencias y necesidades específicas de género en la investigación, práctica y formulación de políticas de salud beneficia a la salud de mujeres y hombres ${ }^{14,15}$.

En definitiva, este estudio ofrece un buen ejemplo de cómo explorar la integración del género en políticas sanitarias y comprobar en qué medida éstas contemplan las desigualdades, discriminaciones, brechas, injusticias y necesidades específicas de género. Medir los avances en mainstreaming de género permitirá identificar qué barreras impiden que el compromiso político se transforme en práctica, ya que es necesario entender las desigualdades que se producen en la formulación de las políticas sanitarias para reducir las desigualdades en la práctica.

12. GUZMAN, Virginia: «La equidad de género como tema de debate y de políticas públicas» en: Memoria del foro internacional sobre cuidadanía, género y reforma del Estado. México, 1997, pp. 89-108.

13. Vives Cases, Carmen. La Violencia Contra Las Mujeres En El Espacio Discursivo Público. Tesis Doctoral, Universidad de Alicante, 2004.

14. DoyAl, Lesley. «Sex, Gender, and Health: The Need for a New Approach.», British Medical Journal, 323:7320 (2001), pp 1061-3.

15. Khouri, Amal J., Carol S. WeISMAn. «Thinking About Women'S Health: The Case for Gender Sensitivity.» Women's health issues, 12:2 (2002), pp.61-65.

Feminismo/s 18, diciembre 2011, pp. 343-351 


\section{Referencias Bibliográficas}

Briones Vozmediano, Erica, Vives Cases, Carmen, Peiró Pérez, Rosana «Sensibilidad de género en los planes nacionales de salud del contexto internacional», Informe SESPAS 2009.

Colomer-Revuelta, Concha, Peiró-Pérez, Rosana, López-Rodríguez, Rosa M.., EsPiga-LÓPEZ Isabel, SÁIZ-MARTíEnZA-ACITORES, Isabel, SORIANO-VILlARROEL, Isabel. «Policies, politics and gender research». Journal of Epidemiology and Community Health, 61, (2007), pp. iil-ii2.

GISPERT, Rosa: «Objetivo 33: Desarrollo De Las Políticas Salud Para Todos.», en Álvarez-Dardet, Carlos, y PeIró, Rosana (eds.) Informe SESPAS 2000.

DoyAl, Lesley. «Sex, Gender, and Health: The Need for a New Approach.», British Medical Journal, 323:7320 (2001), pp 1061-3.

GuZMAN, Virginia: «La equidad de género como tema de debate y de políticas públicas» en: Memoria del foro internacional sobre cuidadanía, género y reforma del Estado. México, 1997, pp. 89-108.

Khouri, Amal J., Carol S. WeISMAn. «Thinking About Women'S Health: The Case for Gender Sensitivity.» Women's health issues, 12:2 (2002), pp.61-65.

ORGANIZACIÓN MUNDIAL DE LA SALUD: Promoción de la salud. Glosario. Ginebra,:

Organización Mundial de la Salud; 1998. ORGANIZACIÓN MUNDIAL DE LA SALUD: Integrating gender perspectives in the work of WHO. WHO gender policy. Ginebra,: Organización Mundial de la Salud; 2002.

ORGANIZACIÓN MUNDIAL DE LA SALUD, A summary of the "so what? Report, Ginebra, Organización Mundial de la Salud, 2005.

ORGANIZACIÓN MUNDIAL DE LA SALUD: WHA 60.25 Resolution. Strategy for integrating gender analysis and actions into the work of WHO. Ginebra, Organización Mundial de la Salud, 2007.

Peiró, Rosana, Alvarez-Dardet, Carlos, Plasencia, Antoni, Borrell, Carmen, Colomer, Concha, Moya, Carmela, PASArín, Maria Isabel, ZAFrA, Eduardo. «Rapid appraisal methodology for 'health for all' policy formulation analysis», Health Policy, 62:3,.(2002), pp. 309-28.

Peiró, Rosana., Vives, Carmen, Álvarez-Dardet, Carlos y R. Mas: «El Análisis De Políticas Con Enfoque De Género Y Salud.», en Carmen Borrell y Lucía ARTAzCOz (ed.), 5a Monografía. Sociedad Española De Epidemiología. Investigación Sobre Género Y Salud,.Barcelona, Sociedad Española de Epidemiología, 2007, pp. 119-37.

Ruiz Cantero, Maria Teresa, Vives Cases, Carmen, Artazcoz, Lucía, Delgado, Ana, García Calvente Maria del Mar, Miqueo, Consuelo, Montero, Isabel, Ortiz, Rocío, Ronda, Elena, Ruiz, Isabel, VAlLs, Carme «A framework 
to analyse gender bias in epidemiological research», Journal of Epidemiology Community Health, 61, (2007), pp. 46-53

SteWART, Donna E., Linda M. Dorado, Natalia Diaz-Granados, Marta Rondon, Javier SAAVEDRA, Jose POSADA-VILla y. Yolanda TORRES. «Examining Gender Equity in Health Policies in a Low- (Peru), Middle- (Colombia), and High(Canada) Income Country in the Americas.», Journal of Public Health Policy, 30: 4, (2009), pp. 439-54.

Vives CASES, Carmen. La Violencia Contra Las Mujeres En El Espacio Discursivo Público. Tesis Doctoral, Universidad de Alicante, 2004. 